\title{
Cardiovascular and respiratory reflexes in the tropical fish, traira (Hoplias malabaricus): $\mathrm{CO}_{2} / \mathrm{pH}$ chemoresponses
}

\author{
Stephen G. Reid ${ }^{\mathrm{a}, *}$, Lena Sundin ${ }^{\mathrm{a}}$, Ana L. Kalinin ${ }^{\mathrm{b}}$, F. Tadeu Rantin ${ }^{\mathrm{b}}$, \\ William K. Milsom ${ }^{a}$ \\ a Department of Zoology, University of British Columbia, 6270 University Blvd., Vancouver BC, Canada V6T $1 Z 4$ \\ ${ }^{\mathrm{b}}$ Department of Physiological Sciences, Federal University of São Carlos, 13565-905 São Carlos SP, Brazil
}

Accepted 9 November 1999

\begin{abstract}
To examine the distribution and physiological role of $\mathrm{CO}_{2} / \mathrm{pH}$-sensitive chemoreceptors in the gills of the tropical fish, traira (Hoplias malabaricus), fish were exposed to acute environmental hypercarbia $\left(1.25,2.5\right.$ and $5.0 \% \mathrm{CO}_{2}$ in air) and subjected to injections of $\mathrm{HCl}$ into the ventral aorta and buccal cavity. This was done before and after selective denervation of branchial branches of the IXth and Xth cranial nerves to various gills arches. Hypercarbia produced a significant decrease in heart rate, a mild hypotension as well as increases in both ventilation rate and ventilation amplitude. The data suggest that the hypercarbic bradycardia and increase in ventilation frequency arise from receptors exclusively within the gills but present on more than the first gill arch, while extra-branchial receptors may also be involved in controlling the increase in ventilation amplitude. With the exception of a decrease in heart rate in response to $\mathrm{HCl}$ injected into the ventral aorta, the acid injections (internal and external) did not mimic the cardiorespiratory responses observed during hypercarbia suggesting that changes in $\mathrm{CO}_{2}$ are more important than changes in $\mathrm{pH}$ in producing cardiorespiratory responses. Finally, the data indicate that chemoreceptors sensitive to $\mathrm{CO}_{2} / \mathrm{pH}$ and to $\mathrm{O}_{2}$ in the gills of this species involved in producing ventilatory responses are distributed in a similar fashion, but that those involved in producing the bradycardia are not. (C) 2000 Elsevier Science B.V. All rights reserved.
\end{abstract}

Keywords: Carbon dioxide, sensitivity, fish gills; Chemoreceptors, fish gills; Control of breathing, $\mathrm{CO}_{2}$ sensitivity, fish gills; Fish, traira (Hoplias malabaricus); Gills, fish, chemoreceptors

\footnotetext{
* Corresponding author. Present address: Department of Physiology, University of Saskatchewan, 107 Wiggins Rd., Saskatoon, Saskatchewan, Canada, S7N 5E5. Tel.: + 1-306966-6550; fax: + 1-306-966-6532.

E-mail address: reidsg@hotmail.com (S.G. Reid)
}

\section{Introduction}

Fish respond to changes in environmental and arterial oxygen and carbon dioxide levels by modifying ventilatory and cardiovascular function in an apparent attempt to minimize arterial blood gas changes (Perry and Wood, 1989; Randall, 
1990). Given that the capacitance of water for $\mathrm{O}_{2}$ is substantially less than the capacitance for $\mathrm{CO}_{2}$, it is generally accepted that fish respond to changes in $\mathrm{O}_{2}$ to a far greater extent than changes in $\mathrm{CO}_{2} / \mathrm{pH}$. None-the-less, many studies have documented the ventilatory responses of fish to environmental hypercarbia, arterial hypercapnia and intra-vascular injections of acid (Dejours, 1973; Randall and Jones, 1973; Janssen and Randall, 1975; Neville, 1979; Smith and Jones, 1982; Thomas and LeRuz, 1982; Aota et al., 1990; Milsom, 1995).

The increase in breathing observed in response to these manipulations has often been attributed to several indirect effects. It has been suggested that hyperventilation may be a result of a decrease in blood oxygen content due to Bohr and Root effects of $\mathrm{CO}_{2} / \mathrm{pH}$ rather than an internal acidosis per se (Smith and Jones, 1982). It has also been suggested (Randall and Taylor, 1991) that circulating catecholamines, released in response to environmental hypercarbia or internal acid injections, are responsible for the increase in breathing. These hypotheses are, however, controversial. There is mounting evidence to suggest that hypercarbia and/or hypercapnia can induce an increase in breathing in a manner that is independent of changes in both arterial oxygen content and plasma catecholamine levels (Heisler et al., 1988; Burleson et al., 1992). For example, goldfish blood exhibits both Bohr and Root effects yet these fish do not hyperventilate during hypercarbia or acid exposure (Dejours, 1973). Additionally, hypercarbia causes hyperventilation in elasmobranchs despite the absence of Bohr and Root effects and the fact that these fish exhibit a very modest hypoxic ventilatory response (Butler and Taylor, 1971). Also, several studies have demonstrated significant respiratory responses to acidosis accompanying hyperoxia or exercise independent of changes in arterial oxygen content or circulating catecholamines (Heisler et al., 1988; Wood et al., 1990). Finally, it has been shown that increasing levels of circulating catecholamines may exert no effect on ventilation or may even induce hypoventilation (Perry et al., 1992).
Given that hypercarbic exposure is also accompanied by the initial development of a respiratory acidosis (Perry and Wood, 1989), it has proven difficult to distinguish between the effects on ventilation of an elevation in arterial $\mathrm{P}_{\mathrm{CO}_{2}}\left(\mathrm{~Pa}_{\mathrm{CO}_{2}}\right)$ and a depression in arterial $\mathrm{pH}(\mathrm{pHa})$. Milsom (1995) suggested that the hypercarbic ventilatory response may be a consequence of the integration of changes in both $\mathrm{Pa}_{\mathrm{CO}_{2}}$ and $\mathrm{pHa}$ acting at separate receptor sites. Based on the work of Heisler et al. (1988), it would appear that the hyperventilatory response may also arise from changes in intracellular $\mathrm{pH}$ at some, as yet unidentified, site.

In addition to ventilatory responses to environmental disturbances such as hypoxia and hypercarbia, there are also changes in cardiovascular function (Taylor, 1992). The heart of teleost fish receives inhibitory parasympathetic innervation from the vagus nerve and excitatory innervation from adrenergic fibers (Taylor, 1992). In many species of fish, environmental hypoxia causes a substantial decrease in heart rate (hypoxic bradycardia; Taylor, 1992; see Sundin et al., 1999 for additional references). The cardiovascular responses to hypercarbia in fish, however, are not well documented although it would appear that hypercarbia too induces bradycardia (Hughes and Shelton, 1962; Burleson et al., 1992).

The respiratory and cardiovascular reflex responses to hypoxia appear to arise primarily, if not exclusively, from receptors within the respiratory passages, gills and other respiratory organs (Smatresk, 1990; Burleson et al., 1992). Within the gills, there appear to be populations of receptors that are externally and internally oriented so as to monitor the water and blood, respectively (see Smatresk, 1990 for review). Although there is growing evidence to suggest that $\mathrm{CO}_{2} / \mathrm{pH}$ receptors are involved in controlling ventilation, the anatomical location, afferent pathways and discharge characteristics of the receptors that mediate these responses remain unknown. There is still no compelling evidence for the existence of central chemoreceptors for $\mathrm{O}_{2}$ or $\mathrm{CO}_{2} / \mathrm{pH}$ in fish (see Burleson et al., 1992 for review).

This study, in conjunction with Sundin et al. (1999), is part of a continuing series of investiga- 
tions designed to identify the location, innervation, stimulus modalities and sensitivity of cardiorespiratory chemoreceptors in a wide range of fish (obligate water breathers, aquatic surface breathers, facultative air breathers and obligate air breathers). Representatives of all of these groups are found in the tropical and sub-tropical regions of Brazil. This study focuses on a hypoxia tolerant obligate water breather, the traira (Rantin et al., 1993). The goals of this study were: (1) to document the respiratory and cardiovascular responses to acute hypercarbia in traira; (2) to determine the location of branchial receptors involved in eliciting these responses; (3) to determine whether there are also extra-branchial receptors involved in eliciting these responses; and (4) to ascertain whether the branchial receptors are oriented to monitor blood (internal), water (external) or both.

\section{Materials and methods}

\subsection{Experimental animals}

Traira (Hoplias malabaricus) are predatory erythrinids found in South America. They are ambush hunters that live amongst roots, large plants and rocks where they can use their colouring to remain hidden before lunging at potential prey. These animals have a cylindrical body and can grow to approximately $50 \mathrm{~cm}$ in length in the wild. The males are more slender and have a straight lateral line while the females are plumper with a convex lateral line. Juveniles are more coloured than adults, being dark green to a rusty brown on top, fading to white on the belly.

Traira $(N=19 ; 269 \pm 17 \mathrm{~g})$ were collected from the Monjolinho Reservoir, Campus of the Federal University of São Carlos. The animals used in these experiments were the same animals used in the companion paper (Sundin et al., 1999); indeed the experiments performed to elucidate the location and roles of branchial $\mathrm{O}_{2}$ receptors and $\mathrm{CO}_{2}$ receptors were performed as a single experimental series. Only the data have been separated for presentation. As a consequence, the companion paper can be consulted for details of the general animal preparation and the procedure used for gill denervation. These procedures can be summarized very briefly as follows. Animals were instrumented with impedance electrodes to measure ventilation, an afferent branchial cannula to measure heart rate and blood pressure and to inject acid solutions internally, and a snout cannula to inject acid solutions into the mouth. Experiments were run on animals with all branchial nerves intact (control; $N=10$ ), with bilateral denervation of the IXth cranial nerve to the gills (IX; $N=6$ ), with bilateral denervation of both the IXth and the pretrematic branch of the Xth cranial nerve to the first gill arch $(\mathrm{G} 1 ; N=10)$ and with complete bilateral denervation of all four gill arches (G4; $N=7$ ).

\subsection{Experimental protocol}

On the day of an experiment, the opercular impedance leads were connected to an impedance converter to measure ventilation frequency (breaths/min) and amplitude (arbitrary units) while the ventral aorta (VA) cannula was connected to a pressure transducer to measure blood pressure $(\mathrm{kPa})$ and heart rate (beats $/ \mathrm{min})$. Additionally, the VA cannula was used to administer internal injections of saline and $\mathrm{HCl}$ (in saline). The external 'snout' cannula was connected to a 5 $\mathrm{ml}$ syringe to facilitate external administration of water or $\mathrm{HCl}$ (in saline or water; see below) into the buccal cavity. Prior to initiating the experimental protocol, the fish were left undisturbed for approximately $30 \mathrm{~min}$ to allow ventilation, heart rate and blood pressure to stabilize.

\subsection{Acid ( $\mathrm{HCl})$ injections}

Following the $30 \mathrm{~min}$ stabilization period, the animal was subjected to a series of internal (via the VA cannula) and external (via the snout cannula) injections of $\mathrm{HCl}$ to stimulate $\mathrm{pH}$ chemoreceptors. Injections of saline (internal) and saline or water (external; see below) served as controls. The injections were administered in the following order: (1) internal saline, (2) internal $\mathrm{HCl}$ $(0.000125,0.00125,0.0125$ and $0.125 \mathrm{mmol} / \mathrm{L}$ in $0.2 \mathrm{ml}$ of saline), (3) external saline/water and (4) 
external acid $(0.125 \mathrm{mmol} / \mathrm{L}$ in $0.3 \mathrm{ml}$ of saline or water). In each case, the cannula was flushed with $0.2 \mathrm{ml}$ of saline (for internal injections) or $0.5 \mathrm{ml}$ of water (for external injections) to ensure complete acid delivery. Following each injection, cardiorespiratory variables were recorded for $3 \mathrm{~min}$. If pre-injection levels of ventilation, blood pressure and heart rate were not restored following the 3 min period, subsequent injections were delayed until all cardiorespiratory variables returned to prior levels or stabilized at a new level.

\subsection{Environmental hypercarbia}

Initial experiments demonstrated that equilibrating the water with $0.1,0.25,0.5$ or $0.75 \% \mathrm{CO}_{2}$ had no effect on ventilation or heart rate/blood pressure. Consequently, the animals were exposed, consecutively, to $5 \mathrm{~min}$ of $1.25,2.5$ and $5.0 \% \mathrm{CO}_{2}$. The animals were then returned to normocapnic conditions and cardiorespiratory variables were monitored for a further $5 \mathrm{~min}$. The water was not pre-equilibrated with each gas, however, and, thus, it is debatable whether the water would have reached full equilibrium with each gas after $5 \mathrm{~min}$. None-the-less, this protocol was chosen because it ultimately produced a response that could, subsequently, be compared after various degrees of branchial denervation. These levels of $\mathrm{CO}_{2}$ were associated with $\mathrm{pH}$ levels ranging from 7.0-6.8 (air) to 5.1-5.0 (5\% $\mathrm{CO}_{2}$ ). Natural waters in the region from which the fish were obtained range from $\mathrm{pH} 5.09$ to 6.34, total dissolved $\mathrm{CO}_{2}$ from 0.82 to $1.79 \mathrm{mM}$ and dissolved $\mathrm{O}_{2}$ from 0.04 to $2.31 \mathrm{mg} / \mathrm{L}$ as a function of depth and season (Mozeto et al., 1997).

\subsection{Data analysis}

Cardiovascular and respiratory variables were analyzed for a $30 \mathrm{sec}$ control period immediately prior to the initiation of hypercarbia and for the last $30 \mathrm{sec}$ of each minute during exposure to the different levels of hypercarbia. Data were analyzed for a $30 \mathrm{sec}$ control period immediately prior to an injection of water, saline or $\mathrm{HCl}$ and at $10 \mathrm{sec}$ intervals for the first minute post injec- tion. During the second and third minute post injection, data were analyzed for a $30 \mathrm{sec}$ period each minute. Although four doses of internal acid were administered (see above), the reported data represent the maximal response obtained from any of the injections. In the initial experiments, saline was used as the vehicle for external $\mathrm{HCl}$ application and some fish appeared very sensitive (expressed as a short period of apnea and bradycardia) to the introduction of saline $(0.9 \% \mathrm{NaCl})$ into the mouth. As such, in these animals, the effect of the prior control injection of saline was subtracted from the $\mathrm{HCl}$ response (see Sundin et al., 1999).

Heart rate, ventral aortic blood pressures and ventilation rates are reported as absolute values. As breathing amplitude was measured in arbitrary units, ventilation amplitude and total ventilation (ventilation frequency $\times$ ventilation amplitude) are reported as a percent change from the control value.

\subsection{Statistical analysis}

The data are reported as the mean \pm 1 standard error of the mean (SEM). All statistical analysis, including determination of normality and variance, was performed with a commercial software package (Sigmastat; Jandel Scientific). The data were analyzed using a two way repeated measures analysis of variance followed by a Student-Newman-Keuls multiple comparison procedure with the data compared to a single control point (i.e. the pre-hypercarbia or pre-injection value). The fiducial limit of significance was set at $5 \%$.

\section{Results}

\subsection{Overall responses to graded hypercarbia with gill innervation intact}

Figure 1 illustrates a typical trace of blood pressure, heart rate and ventilation (rate and amplitude) during exposure to normocarbia (air) and three levels of hypercarbia $(1.25,2.5$ and $5.0 \%$ 
$\mathrm{CO}_{2}$ in air). It is evident from this figure that there is little or no change in breathing, heart rate or blood pressure during exposure to $1.25 \% \mathrm{CO}_{2}$ with subtle changes beginning during the $2.5 \%$ $\mathrm{CO}_{2}$ exposure. When the water is gassed with $5.0 \% \mathrm{CO}_{2}$ in air, however, heart rate begins to decrease significantly whilst both breathing rate and amplitude increase. These changes (bradycardia and hyperventilation) were typical responses of traira to environmental hypercarbia prior to any gill denervation. Note that heart rate returns towards resting values much faster than ventilation upon the return to air.

\subsection{Cardiovascular reflexes}

The effects of hypercarbia on heart rate, both with the gill innervation intact and following the various protocols of denervation (IX, G1 and G4) are illustrated in Fig. 2. With the gill innervation intact, exposure to 1.25 and $2.5 \% \mathrm{CO}_{2}$ did not cause heart rate to vary from the pre-hypercarbic levels. However, when exposed to $5.0 \% \mathrm{CO}_{2}$, heart rate decreased from approximately 57-43 beats/min in the control group. This pronounced bradycardia was also observed during 5.0\% $\mathrm{CO}_{2}$ exposure following denervation of the IX cranial

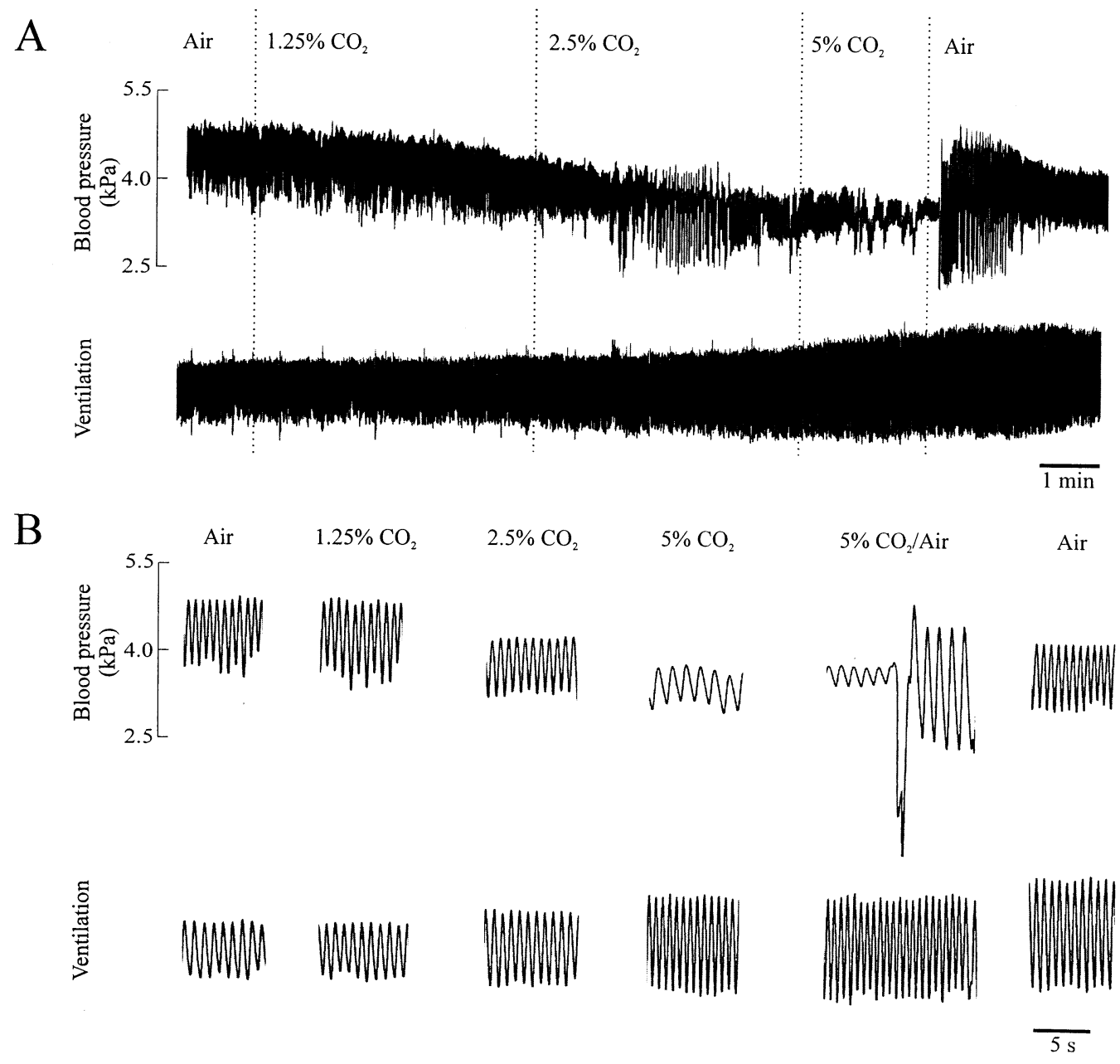

Fig. 1. Representative traces showing cardiovascular and respiratory changes during progressive hypercarbia and recovery. (A) Traces in a compressed form to show the whole response. (B) Traces taken at specific water $\mathrm{CO}_{2}$ levels to display details of the response. 


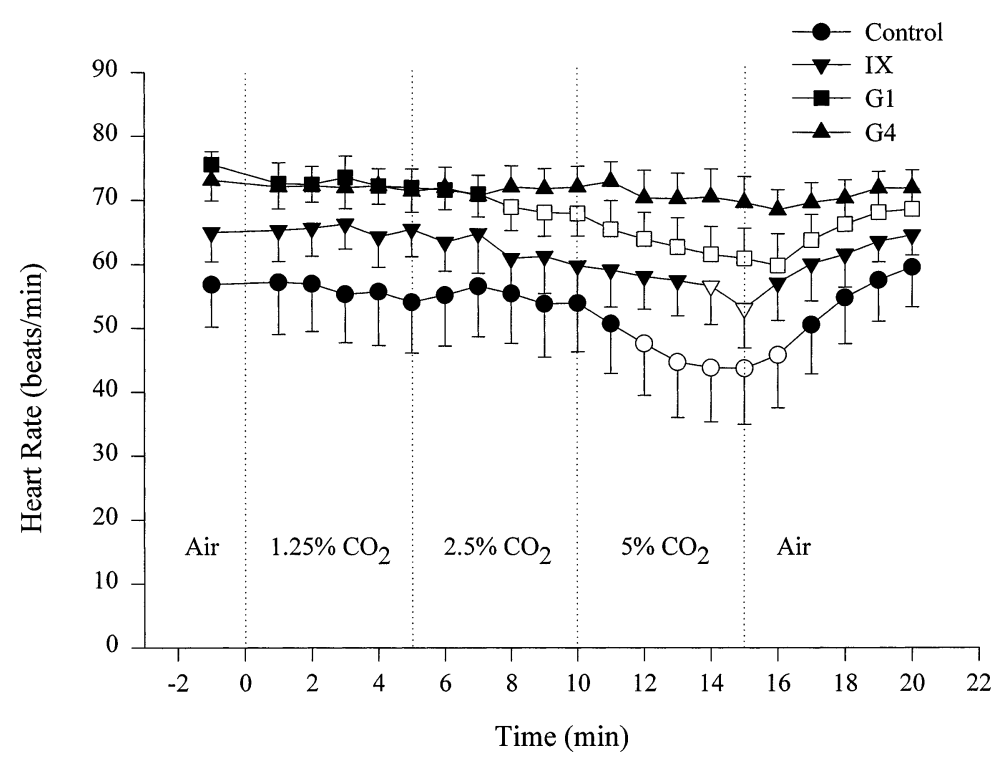

Fig. 2. Heart rate responses to progressive hypercarbia and recovery of intact fish (control; $N=10$ ), with nerve IX sectioned (IX; $N=6$ ), with IX and the pretrematic branch of X to the the 1 st gill arch sectioned ( $\mathrm{G} 1 ; N=10)$, and with IX and branchial branches of $\mathrm{X}$ to all four gill arches sectioned (G4;N=7) (traira have no pseudobranch and therefore the gills of this group were totally denervated). Heart rate showed a significant decrease over time in all groups except in G4 denervated fish. Open symbols are significantly different from starting values.

nerve with heart rate falling from approximately 65-53 beats/min. In the G1 denervated group heart rate also fell, however in this case, the decrease began in response to the $2.5 \% \mathrm{CO}_{2}$, ultimately falling from 75 beats per minute (air) to 59 beats/ min during 5.0\% $\mathrm{CO}_{2}$ exposure. On the other hand, total gill denervation (G4) completely abolished the hypercarbic bradycardia associated with exposure to $5.0 \% \mathrm{CO}_{2}$.

Successive bilateral sectioning of cranial nerves IX and X (i.e. IX, G1 and G4) tended to cause an elevation in resting heart rate (Fig. 2a, air). This increase became statistically significant following total branchial denervation (i.e. G4).

The effects of hypercarbia on ventral aortic blood pressure are illustrated in Fig. 3. In the control group, with gill innervation intact, there was a progressive decrease in blood pressure (from approximately $4.5-3.7 \mathrm{kPa}$ ) commencing during exposure to $2.5 \% \mathrm{CO}_{2}$ and continuing during 5.0\% $\mathrm{CO}_{2}$ exposure. In the other groups, exposure to the various levels of hypercarbia failed to elicit any change in blood pressure despite significant decreases in heart rate in the IX and G1 groups.
Although there was a trend for resting heart rate to increase progressively with successive levels of gill denervation, levels of resting blood pressure tended to decrease progressively with successive levels of denervation (see Fig. 3, prior to the initiation of hypercarbia).

\subsection{Respiratory reflexes}

Figure 4 illustrates the effects of hypercarbia and gill denervation on ventilation rate. In the control group, as well as in the IX and G1 denervated groups, ventilation rate increased during exposure to hypercarbia, with the increases becoming significant during exposure to $5.0 \% \mathrm{CO}_{2}$. On the other hand, ventilation rate in the $\mathrm{G} 4$ group did not increase during exposure to any level of $\mathrm{CO}_{2}$. with levels in this group being, overall, lower than those in the other three groups (Fig. 4b; see below for an explanation of the terminology G4-a and b).

There was a tendency for ventilation amplitude to increase in all of the groups during exposure to hypercarbia with the increase being more pronounced during the $5.0 \% \mathrm{CO}_{2}$ exposure (Fig. 5a). 
These increases were significant in the control group. When analyzing the respiratory amplitude responses to hypercarbia it became evident that not all of the animals in the G4 group responded in a similar fashion. On closer inspection, we noted that five of seven animals exhibited no increase in amplitude in response to any level of hypercarbia (Fig. 5b; G4-a) while two of the seven animals exhibited very brisk responses (Fig. 5b; G4-b).

Figure 6 illustrates the changes in total ventilation during hypercarbia. As was the case with ventilation amplitude, total ventilation increased significantly in control, IX and G1 groups. Again, the data for the G4-a and G4-b groups are presented separately since in the one group of five animals (G4-a, Fig. 6b), the total ventilatory response was eliminated following complete gill denervation while in the other group of two animals (G4-b, Fig. 6b) it was unaffected.

\subsection{Responses to acid injections}

With the exception of a decrease in heart rate in control animals in response to $\mathrm{HCl}$ injected into the ventral aorta, the acid injections (internal or external) were without significant effect.

\section{Discussion}

The results of this study indicate that traira respond to moderate to severe hypercarbia ( $\geq$ $2.5 \% \mathrm{CO}_{2}$ ) with a bradycardia, modest hypotension and increases in both breathing frequency and amplitude. Furthermore, these results indicate that some of the chemoreceptors involved in producing the bradycardia are unique for sensing changes in $\mathrm{CO}_{2} / \mathrm{pH}$ and that their distribution is different from that of the $\mathrm{O}_{2}$-sensitive chemoreceptors that produce similar effects.

\subsection{Receptor sensitivity to changes in $\mathrm{pH}$ versus $\mathrm{CO}_{2}$}

The data raise questions of whether or not the receptors involved in producing the cardiorespiratory responses to environmental hypercarbia respond to changes in $\mathrm{pH}, \mathrm{P}_{\mathrm{CO}_{2}}$, or both. Neither the injections of acid into the water bathing the

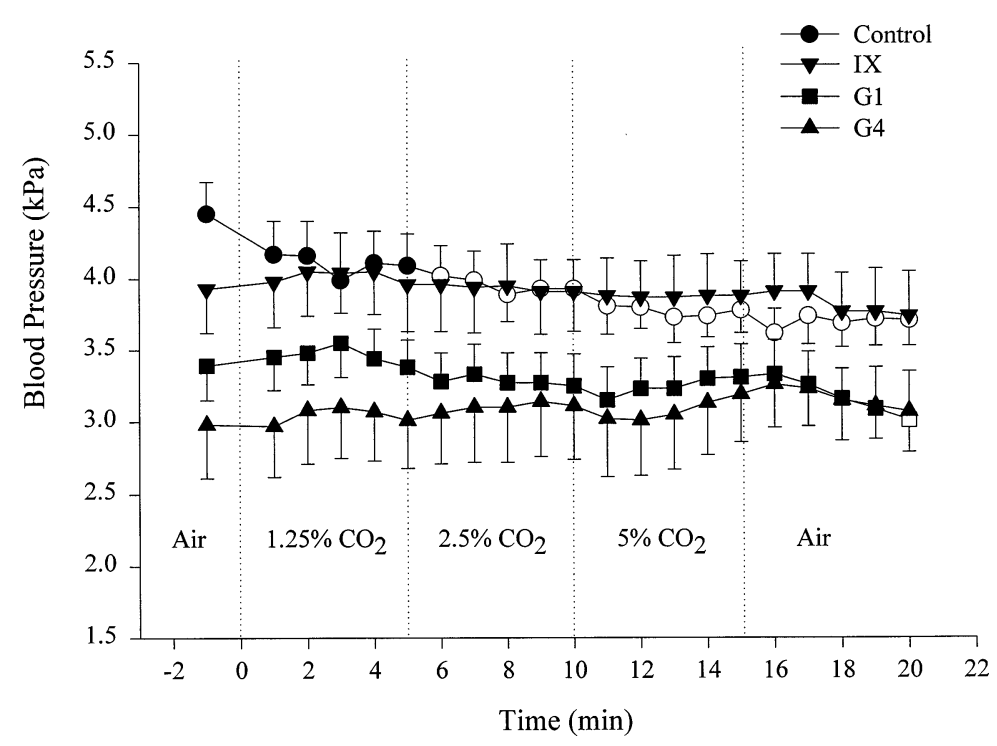

Fig. 3. Prebranchial blood pressure responses to progressive hypercarbia and recovery. Hypercarbia produced a significant decrease in the control group. See Fig. 2 for abbreviations. Open symbols are significantly different from starting values. 


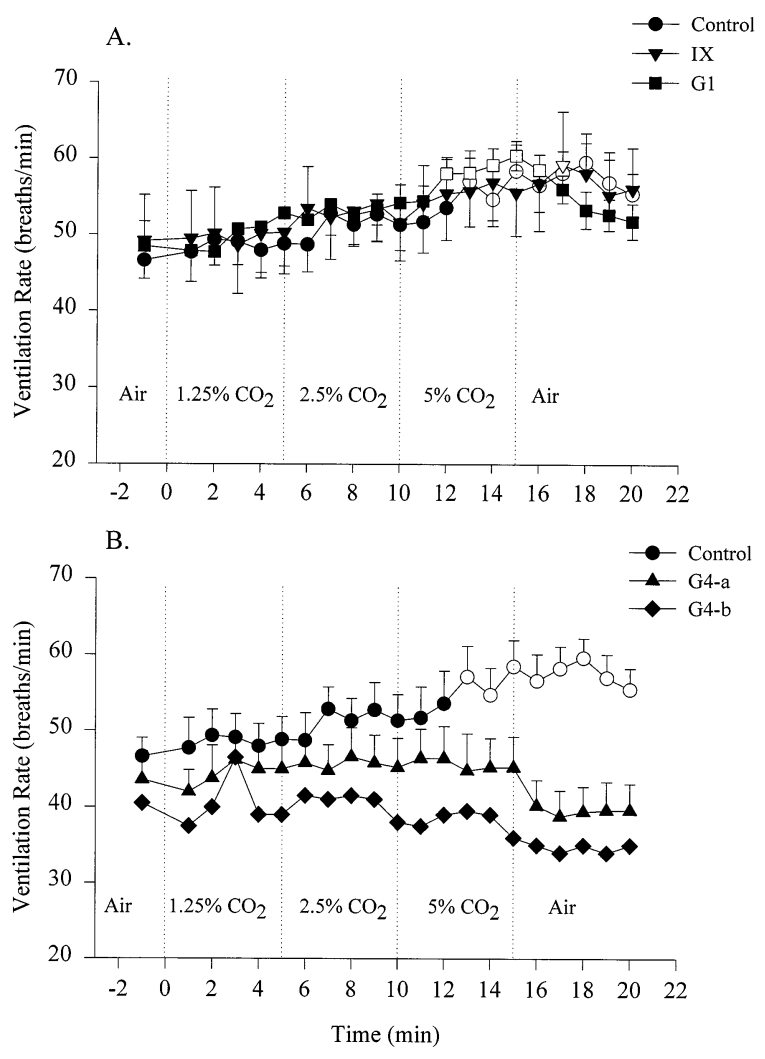

Fig. 4. Ventilation rate responses during hypercarbia and recovery in (A) control, IX and G1 groups and (B) in control, G4-a and G4-b groups. The increases were significant in the control, IX and G1 groups but not the G4 groups (see text for details of G4-a and G4-b groups; see Fig. 2 for abbreviations). Open symbols are significantly different from starting values.

gills nor into the ventral aortic blood had substantial effects on any variable measured. The concentration of the acid solutions used in these experiments were similar to those which produced vigorous responses in other studies (Janssen and Randall, 1975; Neville, 1979; Aota et al., 1990). Studies aimed at separating the effects of $\mathrm{H}^{+}$ions from those of $\mathrm{CO}_{2}$ have suggested that, for salmonids, changes in external or internal $\left[\mathrm{H}^{+}\right]$do not affect ventilation unless accompanied by hypercapnia (Janssen and Randall, 1975; Neville, 1979; Thomas and LeRuz, 1982). On the other hand, attempts to correlate changes in ventilation seen in the skate during hypercarbia to changes in arterial $\mathrm{P}_{\mathrm{CO}_{2}}$, arterial $\mathrm{pH}$, cerebrospinal fluid $\mathrm{pH}$ and calculated brain intracellular $\mathrm{pH}(\mathrm{pHi})$ found no close correlation with any of these variables and concluded that the best correlation was between gill ventilation and either $\mathrm{pHa}$ or $\mathrm{pHi}$ (Wood et al., 1990). Consequently, we feel the data suggest that receptors in traira may be specific for monitoring changes in $\mathrm{P}_{\mathrm{CO}_{2}}$ rather than extracellular $\mathrm{pH}$ per se. Since $\mathrm{CO}_{2}$, but not hydrogen ions, readily cross cell membranes, these receptors may also monitor changes in intracellular $\mathrm{pH}$ which is more sensitive to changes in extracellular $\mathrm{P}_{\mathrm{CO}_{2}}$ than to changes in extracellular $\mathrm{pH}$.

Finally, given that acid injections in the current study were without effect, we are unable to determine the extent to which the receptors in the gills are oriented to sense changes in water versus blood $\mathrm{pH} / \mathrm{P}_{\mathrm{CO}_{2}}$.
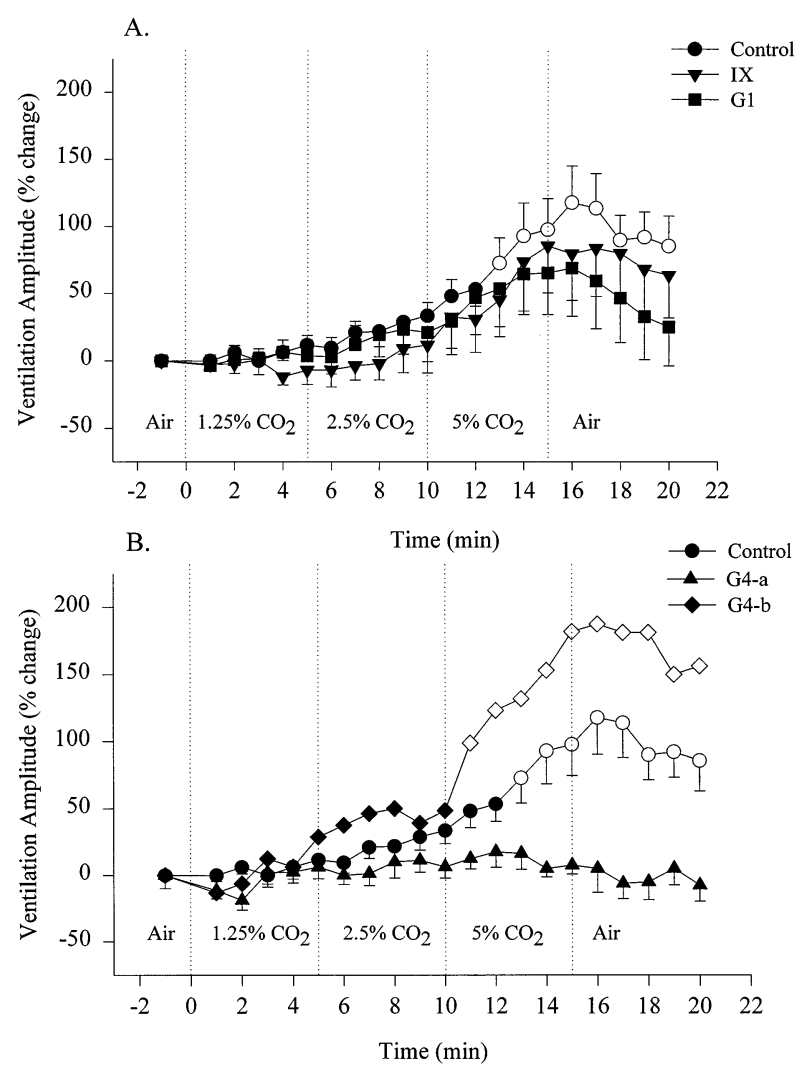

Fig. 5. Changes in ventilation amplitude during progressive hypercarbia and recovery in (A) control, IX and G1 fish and (B) control, G4-a and G4-b fish. The increases were significant in the control, IX and G1 groups. The response was abolished in five of seven animals in the G4 group (G4-a) but not in the other two fish (G4-b). See Fig. 2 for abbreviations. Open symbols are significantly different from starting values. 


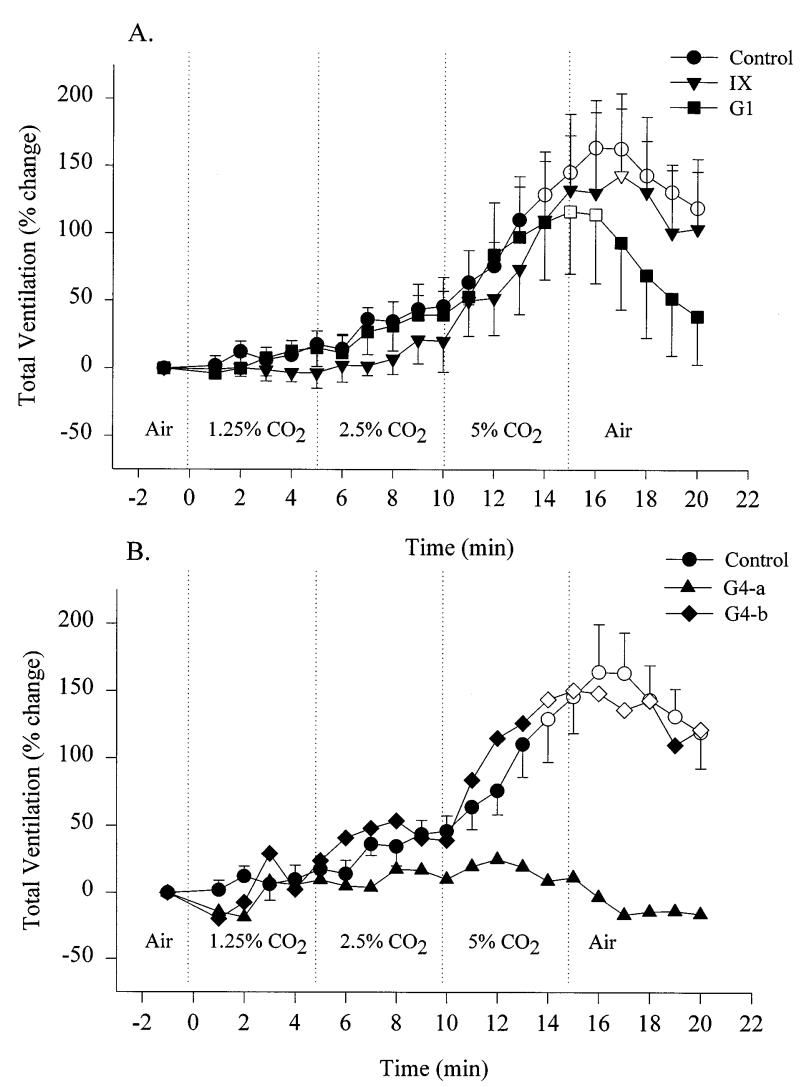

Fig. 6. Changes in total ventilation during progressive hypercarbia and recovery in (A) control, IX and G1 fish and (B) control, G4-a and G4-b fish. The increases were significant in control IX and G1 groups. The response was abolished in five of seven animals in the G4 group (G4-a) but not in the other two fish (G4-b). See Fig. 2 for abbreviations. Open symbols are significantly different from starting values.

\subsection{Receptors mediating the hypercarbic bradycardia}

Numerous studies have described the bradycardia that develops during exposure to environmental hypoxia in fish (see Sundin et al., 1999 for references). On the other hand, we have been unable to find descriptions of the cardiovascular responses to hypercarbia/hypercapnia. A hypercarbic bradycardia has also been observed in another tropical fish, the tambaqui (Colossoma macropomum; Reid et al. unpublished observations) and it is quite possible that this is the typical response of all teleosts.
The results of this study suggest that the receptors mediating the hypercarbic bradycardia in traira are located exclusively within the gills and can be found on more than the first gill arch. Denervation of the IXth cranial nerve alone and both the IXth nerve and the pretrematic branch of the vagus (Xth) nerve to the first gill arch failed to prevent the reflex bradycardia that developed during exposure to environmental hypercarbia. On the other hand, following total arch denervation the bradycardia was abolished. Although these results do not allow us to say whether the receptors are found on all gill arches, we believe this is highly probable.

\subsection{Hypercarbic versus hypoxic bradycardia}

In the companion study (Sundin et al., 1999), the data suggested that the receptors involved in producing the hypoxic bradycardia in this species were internally oriented (i.e. sensed changes in arterial blood) and were located exclusively on the first arch. Given this, the observation that $\mathrm{CO}_{2}$ sensitive receptors involved in producing the hypercarbic bradycardia are found on more than the first gill arch suggests there are separate populations of $\mathrm{O}_{2}$ and $\mathrm{CO}_{2} / \mathrm{pH}$ sensitive receptors with a different distribution across the gill arches. The data do not eliminate the possibility that one common population of receptors on the first gill arch reflexively mediate a bradycardia in response to changes in both $\mathrm{O}_{2}$ and $\mathrm{CO}_{2} / \mathrm{pH}$. They do, however, indicate there are receptors found outside the first gill arch, sensitive to $\mathrm{CO}_{2} / \mathrm{pH}$ alone, which reflexively produce a bradycardia. This raises the possibility that all receptors involved in producing the hypercarbic bradycardia are distinct from those involved in producing the hypoxic bradycardia.

\subsection{Receptors mediating effects on blood pressure}

Exposure to environmental hypercarbia rapidly caused a decrease in blood pressure commencing during exposure to $1.25 \% \mathrm{CO}_{2}$. Unlike the blood pressure response during hypoxia (Sundin et al., 1999), this hypotension did not develop due to a concomitant bradycardia, since heart rate did not 
decrease until exposure to $5 \% \mathrm{CO}_{2}$. Thus, in contrast to a hypoxia-induced vasoconstriction (Sundin et al., 1999), elevated levels of $\mathrm{CO}_{2}$ appear to cause a vasodilation. It is possible that this vasodilation results from a direct dilatory effect of $\mathrm{CO}_{2}$ on vascular smooth muscle, which in mammals functions to control local blood flow. However, it is difficult to ascribe the decrease in blood pressure solely to a direct effect of $\mathrm{CO}_{2}$ on smooth muscle, since blood pressure is maintained during hypercarbia in all of the denervated groups (IX, G1 and G4). Indeed, IX and G1 fish actually maintain blood pressure despite the development of a significant hypercarbic bradycardia.

Since cardiac output was not measured in this study, it is not possible to determine whether blood pressure was maintained in the denervated groups by a compensatory increase in cardiac stroke volume or an increase in systemic vascular resistance. If the latter is true, it suggests that denervation of the first gill arch, or possibly just the IXth nerve to the first arch, removed an active inhibition of excitatory stimuli on systemic vessels which compromises the ability of increases in systemic vascular resistance to maintain blood pressure during hypercarbia. Consistent with this suggestion is the observation that, during environmental hypoxia, the first gill arch is a site of inhibitory signals which blunt the hypoxic ventilatory response in this species (Sundin et al., 1999).

In general, progressive levels of branchial denervation led to a rise in resting heart rate and a decrease in resting blood pressure under normocarbic conditions (Figs. 2 and 3; also see Table 1 in Sundin et al., 1999). For pressure to fall in the face of such large increases in heart rate, either cardiac stroke volume or peripheral resistance must decrease. The progressive increase in resting heart rate, yet decrease in blood pressure, support the suggestion that there is a progressive removal of baroreceptor input with progressive denervation. As such, it appears that under normocarbic conditions in intact fish there is a significant vagal influence on heart rate and that barostatic reflexes accurately maintain blood pressure. This is also true under hypercarbic conditions if the IXth cranial nerve is sectioned bilaterally but not in intact fish where a reflex hypercarbic vasodilation appears sufficient to over-ride the barostatic reflexes and produce hypotension. This is unlike the situation during hypoxia where a systemic vasoconstriction appears to arise from reflex mechanisms triggered by both branchial and extra-branchial receptors (Sundin et al., 1999).

\subsection{Ventilatory responses}

Environmental acidosis, hypercapnia and the arterial acidosis associated with exercise have all been demonstrated to elevate ventilation in fish (e.g. Randall and Jones, 1973; Smith and Jones, 1982; Thomas and LeRuz, 1982; Wood et al., 1990; see review by Milsom, 1995). In this study, environmental hypercarbia produced an increase in both ventilation frequency and amplitude. Previous studies on teleosts have also demonstrated that both ventilation rate and amplitude increase during hypercarbia (dogfish; Heisler et al., 1988; skate; Wood et al., 1990; trout; Kinkead and Perry, 1991; Gilmour and Perry, 1994). As such, the hyperventilatory response of the traira to hypercarbia, appears to be consistent with previous studies.

On the other hand, the levels of $\mathrm{CO}_{2}$ required to obtain hypercarbic responses (i.e. bradycardia, hypotension and hyperventilation) in our study are substantially greater than in previous studies on temperate species. Thus while rainbow trout respond to a change in $\mathrm{Pw}_{\mathrm{CO}_{2}}$ of approximately 5 torr (Kinkead and Perry, 1991; Gilmour and Perry, 1994), traira did not respond until 5\% $\mathrm{CO}_{2}$ had been added to the water which corresponds to a $\mathrm{Pw}_{\mathrm{CO}_{2}}$ of approximately 38 torr. However, given that neotropical fish such as the traira live in extremely acidic waters $(\mathrm{pH} 3.5-5.0)$, it is not surprising that such levels of $\mathrm{CO}_{2}$ were required to elicit cardiovascular and respiratory responses. The natural waters in the region from which these fish were obtained are typically supersaturated with free carbon dioxide with respect to the atmosphere and range from $\mathrm{pH} 5.09$ to 6.34 , total dissolved $\mathrm{CO}_{2}$ from 0.82 to $1.79 \mathrm{mM}$ and dissolved $\mathrm{O}_{2}$ from 0.04 to $2.31 \mathrm{mg} / \mathrm{L}$ as a function of depth and season (Mozeto et al., 1997). 


\subsection{Receptors mediating the increase in ventilation rate during hypercarbia}

Ventilation rate increased during hypercarbia in intact animals as well as in animals following IX and $\mathrm{G} 1$ denervation. It did not increase following total gill arch denervation indicating that the increase in ventilation frequency during hypercarbia was mediated by receptors present exclusively in the gills and present on more than just the first gill arch.

\subsection{Receptors mediating the increase in ventilation amplitude during hypercarbia}

The effects of progressive gill denervation on the increase in ventilation amplitude produced by hypercarbia are more difficult to interpret since not all fish responded in a similar fashion. While all fish exhibited similar increases in ventilation amplitude in the control, IX and G1 groups, this was not the case in the G4 group. In this group, the increase in ventilation amplitude was abolished in five of seven animals but remained unchanged in the other two animals. It is unlikely that the branchial nerves were not completely sectioned in the latter two fish since all branches were accounted for post-mortem and were indeed completely sectioned. Furthermore, the hypercarbic bradycardia and the increase in ventilation frequency, both of which required complete gill denervation to abolish in the other five fish, were also abolished in these two animals. At present it is difficult to resolve this discrepancy. Taken together, the data from the group of five G4 animals that did not increase ventilation amplitude during hypercarbia suggest that the receptors involved in reflexively elevating ventilation amplitude are confined to the gills while the increase in ventilation amplitude in the additional two animals must clearly be triggered from other sites.

If extra-branchial receptors are involved in mediating the increase in ventilation amplitude in response to hypercarbia in the two G4b fish, the same putative sites suggested for the extrabranchial receptors contributing to the increase in ventilation amplitude during hypoxia are likely candidates. These include the orobranchial cavity innervated by cranial nerves $\mathrm{V}$ (trigeminal) and VII (facial) (e.g. Butler et al., 1977) and the central nervous system (Saunders and Sutterlin, 1971). However, all attempts to evoke a ventilatory response in fish by central stimulation with acid or alkaline perfusates have failed (see Hedrick et al., 1991 for review). It has also been suggested that catecholamines released during hypercarbia may play a role in the ventilatory response to hypercarbia (Randall and Taylor, 1991) but this suggestion remains controversial (Perry et al., 1992).

\subsection{Hypercarbic versus hypoxic hyperventilation}

The receptors involved in producing the increases in both breathing frequency and amplitude appear to have a similar distribution to those involved in producing the ventilatory responses to hypoxia (Sundin et al., 1999). This raises several possibilities. One is that both sets of reflexes are initiated by the same receptors which can be stimulated by changes in both $\mathrm{O}_{2}$ and $\mathrm{CO}_{2} / \mathrm{pH}$ per se, as is the case in the mammalian carotid body (González et al., 1994). Another possibility is that the same receptors are involved in producing both hypoxic and hypercarbic ventilatory responses, but, the response to changes in $\mathrm{CO}_{2} / \mathrm{pH}$ is a consequence of their effects on the $\mathrm{O}_{2}$ carrying capacity of the blood rather than on $\mathrm{CO}_{2} / \mathrm{pH}$ sensing mechanism. If the ventilatory responses to hypercarbia are acting via a depression of blood oxygen carrying capacity, then the chemoreceptors that activate these reflexes must be sensitive to changes in oxygen content as is the case in the mammalian aortic body (Lahiri et al., 1981). Since the data suggest that the ventilatory responses to hypoxia arise primarily from externally-oriented receptors (Sundin et al., 1999), however, it is unlikely that these effects can be due solely to a decrease in blood oxygen content. Yet another possibility, based on the fact there appear to be receptors found outside the first gill arch involved in producing the hypercarbic bradycardia that respond to changes in $\mathrm{CO}_{2} / \mathrm{pH}$ only, there may be separate receptors involved in hypoxic and hypercarbic ventilatory responses with similar distributions. 


\section{Summary}

During acute exposure to hypercarbia in traira, heart rate and blood pressure decrease and breathing rate and amplitude both increase. The receptors mediating the bradycardia and increase in ventilation frequency are located exclusively within the gills and on more than the first gill arch. While this may also be true of the receptors mediating the increase in ventilation amplitude, there is some evidence to indicate that this increase may also involve extra-branchial receptors. Finally the data indicate that while some of the receptors involved in the cardiorespiratory responses to hypercarbia may be the same as those involved in the responses to hypoxia, some are clearly unique.

\section{Acknowledgements}

This study was supported by the Natural Sciences and Engineering Research Council (NSERC) of Canada, the Brazilian National Research Council for Development of Sciences and Technology (CNPq), Fundação de Amparo à Pesquisa do Estado de São Paulo (FAPESP), the Isaak Walton Killam Foundation, the Swedish Foundation for International Cooperation in Research and Higher Education, the Wenner-Gren Center Foundation and the Royal Society of Arts and Sciences in Göteborg.

\section{References}

Aota, S., Holmgren, K.D., Gallaugher, P., Randall, D.J., 1990. A possible role for catecholamines in the ventilatory responses associated with internal acidosis or external hypoxia in rainbow trout, Oncorhynchus mykiss. J. Exp. Biol. 151, 57-70.

Burleson, M.L, Smatresk, N.J., Milsom, W.K., 1992. Afferent inputs associated with cardiovascular control in fish. In: Hoar, W.S., Randall, D.J. (Eds.), Fish Physiology. Academic Press, New York, pp. 389-426.

Butler, P.J., Taylor, E.W., 1971. Responses of the dogfish (Scyliorhinus canicula L.) to slowly induced and rapidly induced hypoxia. Comp. Biochem. Physiol. A 39, 307-323.

Butler, P.J., Taylor, E.W., Short, S., 1977. The effect of sectioning cranial nerves V, VII, IX and X on the cardiac responses of the dogfish Scyliorhinus canicula to environmental hypoxia. J. Exp. Biol. 69, 233-245.

Dejours, P., 1973. Problems of control of breathing in fishes. In: Bolis, L., Schmidt-Nielsen, K., Maddrell, S.H.P. (Eds.), Comparative Physiology. Elsevier/North-Holland, Amsterdam, pp. 117-133.

Gilmour, K.M., Perry, S.F., 1994. The effects of hypoxia, hyperoxia or hypercapnia on the acid-base disequilibrium in the arterial blood of rainbow trout. J. Exp. Biol. 192, 269-284.

González, C., Almarez, L., Obeso, A., Rigual, R., 1994. Carotid body chemoreceptors: from natural stimuli to sensory discharges. Physiol. Rev. 74 (4), 829-898.

Hedrick, M.S., Burleson, M.L., Jones, D.R., Milsom, W.K., 1991. An examination of central chemosensitivity in an air-breathing fish (Amia calva). J. Exp. Biol. 155, 165-174.

Heisler, N., Toews, D.P., Holeton, G.F., 1988. Regulation of ventilation and acid-base status in the elasmobranch Scyliorhinus stellaris during hyperoxia-induced hyper carbia. Respir. Physiol. 71, 227-246.

Hughes, G.M., Shelton, G., 1962. Respiratory mechanisms and their nervous control in fish. Adv. Comp. Physiol. Biochem. 1, 275-364.

Janssen, R.G., Randall, D.J., 1975. The effects of changes in $\mathrm{pH}$ and $\mathrm{P}_{\mathrm{CO}_{2}}$ in blood and water on breathing in rainbow trout, Salmo gairdneri. Respir. Physiol. 25, 235-245.

Kinkead, R., Perry, S.F., 1991. The effects of catecholamines on ventilation in rainbow trout during hypoxia or hypercapnia. Respir. Physiol. 84, 77-92.

Lahiri, S., Mulligan, E., Nishino, T., Mokashi, A., Davies, R.O., 1981. Relative responses of aortic body and carotid body chemoreceptors to carboxyhemoglobinemia. J. Appl. Physiol. 50, 580-586.

Milsom, W.K., 1995. Regulation of respiration in lower vertebrates: role of $\mathrm{CO}_{2} / \mathrm{pH}$ receptors. In: Advances in Comparative and Environmental Physiology, vol. 21. Springer, Berlin, pp. 6-104.

Mozeto, A.A., Krusche, A.V., Luccas, P.O., 1997. Aspectos do ciclo biogeoquimico do enoxfre em uma lagoa marginal da area alagavel do Rio Moji-Estacao Ecologica de Jatai, Luiz Antonio, SP. Geochem. Brail 11, 231-241.

Neville, C.M., 1979. Ventilatory responses of rainbow trout (Salmo gairdneri) to increased $\mathrm{H}^{+}$ion concentration in blood and water. Comp. Biochem. Physiol. A 63, 373-376.

Perry, S.F., Wood, C.M., 1989. Control and coordination of gas transfer in fish. Can. J. Zool. 67, 2961-2970.

Perry, S.F., Kinkead, R., Fritsche, R., 1992. Are circulating catecholamines involved in the control of breathing by fishes? Rev. Fish Biol. Fisheries 2, 65-83.

Randall, D.J., Jones, D.R., 1973. The effects of deaffereniation of the pseudobranch on the respiratory response to hypoxia and hyperoxia in the trout (Salmo gairdneri). Respir. Physiol. 17, 291-302.

Randall, D.J., 1990. Control and co-ordination of gas exchange in water breathers. In: Advances in Comparative and Environmental Physiology, vol. 6. Springer, Berlin, pp. $253-278$. 
Randall, D.J, Taylor, E.W., 1991. Evidence of a role for catecholamines in the control of breathing in fish. Rev. Fish Biol. Fisheries 1, 139-157.

Rantin, F.T., Glass, M.L., Kalinin, A.L., Verzola, R.M.M., Fernandes, M.N., 1993. Cardio-respiratory responses in two ecologically distinct erythrinids (Hoplias malabaricus and Hoplias lacerdae) exposed to graded environmental hypoxia. Environ. Biol. Fishes 36, 93-97.

Saunders, R.L., Sutterlin, A.M., 1971. Cardiac and respiratory responses to hypoxia in The sea raven, Hemitripterus americanus, and an investigation of possible control mechanisms. J. Fish. Res. Bd. Can. 28, 491-503.

Smatresk, N.J., 1990. Chemoreceptor modulation of endogenous respiratory rhythms in vertebrates. Am. J. Physiol. 259, R887-R897.

Smith, F.M., Jones, D.R., 1982. The effect of changes in blood oxygen carrying capacity on ventilation volume in the rainbow trout (Salmo gairdneri). J. Exp. Biol. 97, 325334.

Sundin, L., Reid, S.G., Kalinin, A., Rantin, T., Milsom, W.K., 1999. Regulation of cardiorespiratory reflexes in the Traira (Hoplias malabaricus): I. The role of O2 chemoreceptors. Respir. Physiol. 116, 181-199.

Taylor, E.W., 1992. Nervous control of the heart and cardiorespiratory interactions. In: Randall, D.J., Hoar, W.S. (Eds.), Fish Physiology, vol. XIIB. Academic Press, New York, pp. 343-387.

Thomas, S., LeRuz, H., 1982. A continuous study of rapid changes in blood acid-base status of trout during variations of water $\mathrm{P}_{\mathrm{CO}_{2}}$. J. Comp. Physiol. B 148, 123-130.

Wood, C.M., Turner, J.D., Munger, R.S., Graham, M.S., 1990. Control of ventilation in the hypercarbic skate Raja oscellata. II. Cerebrospinal fluid and intracellular $\mathrm{pH}$ in the brain and other tissues. Respir. Physiol. 80, 279-298. 